\title{
Genome-wide sequence identification and expression analysis of ARF family in sugar beet (Beta vulgaris L.) under salinity stresses
}

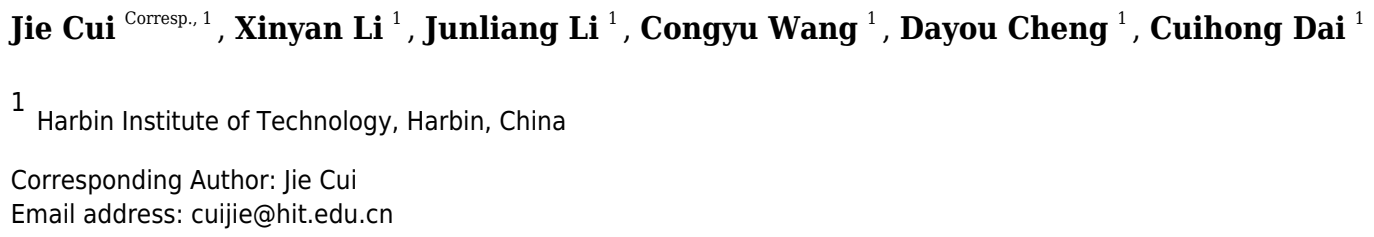

Auxin response factor(ARF) proteins respond to biological and abiotic stresses and play important roles in regulating plant growth and development. In this study, based on the genome-wide database of sugar beet, 16 BvARF proteins were identified. A detailed investigation into the BvARF family is performed, including analysis of the conserved domains, chromosomal locations, phylogeny, exon-intron structure, conserved motifs, subcellular localization, gene ontology (GO) annotations and expression profiles of BvARF under salt-tolerant condition. The majority of BvARF proteins contain B3 domain, AUX_RESP domain and AUX/IAA domain and a few lacked of AUX/IAA domain. Phylogenetic analysis suggests that the 16 BvARF proteins are clustered into 6 groups. Expression profile analysis shows that most of these BVARF genes in sugar beet under salinity stress were up-regulated or down-regulated to varying degrees and 9 of the BvARF genes changed significantly. They were thought to have a significant response to salinity stress. The current study provides basic information for the BVARF genes and will pave the way for further studies on the roles of BvARF genes in regulating sugar beet's growth, development and responses to salinity stress. 
1 Genome-wide sequence identification and expression analysis of ARF

2

\title{
family in sugar beet (Beta vulgaris L.) under salinity stresses
}

\author{
Jie Cui*1, Xinyan Li, Junliang Li, Congyu Wang, Dayou Cheng, Cuihong Dai
}

Auxin response factor(ARF) proteins respond to biological and abiotic stresses and play important roles in regulating plant growth and development. In this study, based on the genome-wide database of sugar beet, 16 BvARF proteins were identified. A detailed investigation into the BvARF family is performed, including analysis of the conserved domains, chromosomal locations, phylogeny, exon-intron structure, conserved motifs, subcellular localization, gene ontology $(\mathrm{GO})$ annotations and expression profiles of BvARF under salt-tolerant condition. The majority of BvARF proteins contain B3 domain, AUX_RESP domain and AUX/IAA domain and a few lacked of AUX/IAA domain. Phylogenetic analysis suggests that the 16 BvARF proteins are clustered into 6 groups. Expression profile analysis shows that most of these BvARF genes in sugar beet under salinity stress were up-regulated or downregulated to varying degrees and 9 of the BvARF genes changed significantly. They were thought to have a significant response to salinity stress. The current study provides basic information for the BvARF genes and will pave the way for further studies on the roles of BvARF genes in regulating sugar beet's growth, development and responses to salinity stress.

Key words: Sugar beet; ARF; salinity stress; bioinformation

\section{Introduction}

$\mathrm{ARF}$ are transcription factors that activate or inhibit the expression of auxin-response genes by binding to specific positions in the promoter region of auxin-responsive genes, thereby regulating plant growth and development, including vascular elongation, cell division, apical dominance, flowering, fruit development (Chandler, 2016; Fleming, 2006; Kumar, Tyagi \& Sharma, 2011; Li et al., 2016; Ljung, 2013; Su et al., 2014), and responding to abiotic stresses. Most of the ARF proteins consist three conserved domains: the N-terminus DNA binding

\footnotetext{
${ }^{1}$ Jie Cui, Associate Professor of Harbin Institute of Technology, Mingde Building A602, 92 Xidazhi Street, Nangang District, Harbin, Heilongjiang Province, E-mail:cuijie@hit.edu.cn, Tel:13100888032
} 
domain(DBD), the middle region(MR), and the $\mathrm{C}$-terminus domain(PB1). Crystal structures of the DBDs of ARF1 and ARF5 highlight the presence of three different subdomains: a B3 subdomain involved in the recognition of the ARF-specific AuxRE DNA motif, a dimerization domain (DD) allowing ARF dimerization, and a Tudor-like ancillary domain (AD) of unknown function which might be involved in an interaction with the $\mathrm{DD}$ (Roosjen, Paque \& Weijers, 2018). The B3 DNA binding domain is capable of binding to a TGTCTC/GAGACA site in the promoter region of the target genes. And ARF proteins homodimerize through their DD, which were also called AUX_RESP domain in ARF, by hydrophobic interactions(Boer et al., 2014). The MR domain has an activation domain (AD) or a repression domain (RD) activity, thereby having an effect of activating or inhibiting transcription. The PB1 domain is the region of homologous dimerization and heterologous dimerization of ARFs or AUX/IAA proteins and ARF (Yu et al., 2014).

Since the first Arabidopsis ARF gene, ARF1, was cloned and its function investigated (Ulmasov, Hagen \& Guilfoyle, 1997), the research on ARF of Arabidopsis has entered into the molecular mechanism of regulating plant growth. In recent years, Krogan and others had identified the direct regulation relationship between genes that play a major role in auxin signaling and trafficking (Krogan et al., 2016). Studies by Liu et al. showed that ARF 3 promotes flower meristem formation by inhibiting WUS expression (Liu et al., 2014). Zhao demonstrated that the transcription factor ARF2 modulates the expression of the $\mathrm{K}^{+}$transporter gene HAK5 in Arabidopsis thaliana, and found that AtARF2 responds to low potassium stress(Zhao et al., 2016). Zhang studied in AtARF5 and found that it could up-regulate STOMAGEN significantly to regulate stomatal development (Zhang et al., 2014). Report of Li et al. showed that the transcription factors ARF7 and ARF19 are not only important for auxin signaling, but also play critical roles for Arabidopsis to respond to ethylene. It may indicated that AtARF7 and AtARF19 may be involved in the interaction of the ethylene pathway and the auxin pathway (Li, Dai \& Zhao, 2006). With the deepening of research on model plant ARF, the research on ARF of other plants has also started. There are articles on the whole gene analysis of plant ARF such as maize 
51 (Xing et al., 2011), sweet orange (Li et al., 2015), rice (Wang et al., 2007), banana (Hu et al., 2015b) and grape (Wan et al., 2014). In these studies, ARF were divided into 6 classes according to their homology to Arabidopsis thaliana ARF. Some members of these 6 classes are able to respond to one or more abiotic stresses. It is worth noting that in the study of physic nut ARF (Tang et al., 2018), seven ARFs such as JcARF2, JcARF11, JcARF14 and JcARF15 showed significant changes in expression under salt stress conditions. These JcARFs belong to different 5 classes, so it is speculated that ARF may be generally involved in the response of salt stress. And study in tea trees (Xu et al., 2016) have shown that ARF members in roots and shoots of tea plants respond to salt stress, and CsARF6 shows significant up-regulation under different stress conditions, while CsARF2-1 and CsARF11 show significant down-regulation. It is speculated that CsARF members may participate in the regulation of salt tolerance through different ways.

Sugar beet is a biennial herb of Chenopodiaceae. It is an important sugar-producing crop and widely used in food, sugar production, feed, etc. The yield and quality of sugar beet are susceptible to various biologic and abiotic stresses, especially the soil salinity. In China, the sugar beet production area and the saline land overlap in a large area. Even though sugar beet is a salt-tolerant plant, it is limited in extent. The high salinity of the land not only decreases the suitable planting area of sugar beet but also reduces the yield per unit area. In general, the study of salt-tolerant transcription factors contributes to the cultivation of salt-tolerant related sugar beets and lays the foundation for increasing total sugar beet yield. ARF genes participate in responses to abiotic stresses (Qi et al., 2012; Shen et al., 2013), however, there is no genomewide analysis for BvARF. Dohm et al. constructed the whole genome of sugar beet. The physical map and sequence of the whole genome of sugar beet (Dohm et al., 2012; Dohm et al., 2014) make it possible to study the salt tolerance of sugar beet from the genetic level. Based on the whole genome database of sugar beet, the genome-wide analysis of BvARF was carried out by bioinformatics method, and the ARF related to salt treatment was identified, which provide a basis of breeding salt-tolerant sugar beet varieties. 


\section{Materials and methods}

\section{Materials}

The research materials used in this experiment for salinity stress were "O68" strain, which was the salt-tolerant sugar beet strain selected by the laboratory(Shi et al., 2008). In the sugar beet seedling stage, the sugar beet seeds were first soaked in running water for $12 \mathrm{~h}$ and then sown into the wet sponge which was placed in the incubator for 2 days (dark, $24{ }^{\circ} \mathrm{C}$ ). Sugar beet seedlings were taken out after they germinated and placed in the culture pot at $22 \pm 1{ }^{\circ} \mathrm{C}$ with 16 $\mathrm{h} / \mathrm{d}$ supplemental lighting, followed by $8 \mathrm{~h} / \mathrm{d}$ darkness, and cultured with clear water until they grow a third pair of true leaves, then they were treated with salinity stress. The salt stress conditions were that the nutrient solution was replaced with a $300 \mathrm{mM} \mathrm{NaCl}$ solution for 24 hours, the remaining conditions were unchanged, and the control plants were set at the same time without salt treatment. After the stress treatment, leaves and roots were collected. And they were wrapped in tin foil, quickly placed in liquid nitrogen for precooling. The frozen samples were uniformly stored in the ultra-low temperature refrigerator $\left(-80^{\circ} \mathrm{C}\right)$.

BvARF identification in sugar beet

The whole genome data of sugar beet was published by Max Planck Institute for Molecular Genetics in 2014 (http://bvseq.molgen.mpg.de/index.shtml). And the assembly RefBeet-1.1 of KWS2320 was downloaded for bioinformation research. It includes functional annotation, genome sequence, transcript sequences, and protein sequences etc. ARF proteins conserved domain (PF06507) on Pfam were used as a seed sequence to search the whole genome data, and e-value $<1 \mathrm{e}^{-5}$ was set on HMMER (http://www.hmmer.org/). Pfam online tool performed a conserved domain analysis of candidate BvARF protein sequences (Finn et al., 2016), screening protein sequences containing the B3 domain (PF02362), AUX_RESP domain (PF06507) and AUX/IAA (PF02309) domains to remove redundant proteins, and remainders were considered to be BvARF proteins. DNAMAN7.0 was used for multiple sequences alignment of the full-length coding sequence of BvARF proteins, and the conserved domains in the BvARF protein 
103

104

105

106

107

108

109

110

111

112

113

114

115

116

117

118

119

120

121

122

123

124

125

126

127

128

sequences were identified by Weblogo2.8.2 (http://weblogo.berkeley.edu/logo.cgi).

Bioinformatics analysis of BvARF family

Molecular weight and isoelectric point of BvARF proteins were predicted by ExPASY (https://web.expasy.org/protparam/). MapInspect was used to map the position of the BvARF genes on chromosomes. Exon and intron structures of the BvARF genes were analyzed by GSDS (http://gsds1.cbi.pku.edu.cn/) (Hu et al., 2015a). ClustalX was used to carry out a multiple sequence alignment of BvARF proteins(Larkin et al., 2007). A phylogenetic tree of BvARF family and AtARF family and a phylogenetic tree of BvARF family were constructed by the neighbor-joining with bootstrap replicate set to 1000 , and the other parameters default. And the protein sequences of AtARF for comparison were provided in Supplemental File 1. BvARF proteins were submitted to MEME (http://meme-suite.org/tools/meme) in the order of homology and subjected to motif analysis(Bailey et al., 2006), in which the number of expected motifs was set to 20 , and the rest parameters were all default values. Subcellular localization of BvARF proteins was predicted by using CELLO (http://cello.life.nctu.edu.tw/). Gene Ontology (GO) categories of genes were obtained from PlantTFDB (http://plantregmap.cbi.pku.edu.cn/download.php\#go-annotation), and GO level 2 of BvARF genes were visualized with WEGO (http://wego.genomics.org.cn/). And the GO annotation numbers of BvARF were provided in Supplemental File 2.

Expression analysis of BvARF under salinity stress

Total RNA was isolated from the aforementioned sugar beet samples using MiniBEST Plant RNA Extraction Kit (TaKaRa, Japan) and reverse transcribed into cDNA using High Capacity cDNA Reverse Transcription Kit (applied biosystems, USA). The patterns of expression of these genes under normal growth conditions and in response to salinity stresses were analyzed by quantitative real-time PCR (qRT-PCR). Primers for qPCR were designed using Primer 5 and NCBI Primer-BLAST, synthesized by Huada Gene Co., Ltd., and the primer sequences are shown in Tab 1. The ICDH (Isocitrate dehydrogenase) gene was used as an internal control. 
For quantitative analysis using the CFX96 Real-Time System (BIO-RAD, USA), and iTaq Universal SYBR Green Supermix kit (BIO-RAD, USA) was used with ICDH as the housekeeping gene. The reaction system was $10 \mu \mathrm{L}$, in which the dye was $5 \mu \mathrm{L}$, the ddH2O was $3.4 \mu \mathrm{l}$, the forward primer and reverse primers were each $0.4 \mu \mathrm{L}$, and the cDNA was $0.8 \mu \mathrm{L}$, and each treatment was repeated three times. Data analysis was performed by calculating $2^{-\Delta \Delta \mathrm{T}}$, and the relative expression amount of each gene was expressed by mean \pm standard deviation. Results

Identification of BvARF family members

Seventeen candidate BvARF proteins were obtained by using PF06507 as a seed sequence to perform HMMER alignment in the sugar beet genome database. And the sequences of 17 candidate BvARF proteins were analyzed by Pfam for conserved domain, and the proteins containing no specific domains of ARF were removed. A total of 16 BvARF proteins were identified, as shown in Fig 1.

It can be seen from the figure that all BvARF have a $\mathrm{C}$-terminal $\mathrm{B} 3$ binding domain and an intermediate AUX_RESP domain, while the AUX/IAA domain exists only in 11 BvARF except for opag, okdq, ghtj, jrpi, and eqms.

From the comparison results of DNAMAN7.0, the B3 DNA binding domain is highly conserved and occasionally modified. The AUX_RESR domain is not as conserved as B3, the AUX/IAA domain is the least conserved and in short length, as shown in Fig 2.

In the 16 BvARF proteins, gcik.ti and gcik.t2 were both translation products of transcript variants of BvARF gene gcik. And the efdx.t1 and efdx.t2 were both translation products of transcript variants of BvARF gene efdx. Therefore, a total of 14 BvARF gene loci were obtained and indicated by the name in the sugar beet genome-wide database.

The 16 BvARF proteins were blastp aligned with the NCBI database and all found to be members of the BvARF family, as shown in Tab 2. No more new BvARF was identified. 
154

155

156

157

158

159

160

161

162

163

164

165

166

167

168

169

170

171

172

173

174

175

176

177

178

Descriptions in NCBI suggest that jrpi, opag and zzhs may have isoforms.

Analysis of physicochemical properties of BvARF proteins

Sequence analysis showed that the average length of the coding region of ARF gene was 2390 bp (1734-3429 bp), the average number of amino acids encoding proteins was 796 (577$1142)$, and the average molecular weight predicted by ExPASY was $88.7 \mathrm{kDa}(64060.51$ 127711.63 Da), the isoelectric point averages 6.13 (5.21-7.9), as shown in Tab 3.

\section{Chromosomal localization of BvARF gene}

The location analysis showed that 11 BvARF genes were distributed in 8 chromosomes except chromosome 3 . There are 2 BvARF on chromosomes 1, 8, and 9, and one on chromosomes 2, 4, 5, 6, and 7, as shown in Fig 3. The location information of other three genes hgze, jrpi, orwr is unknown in the database, that may be caused by these BvARF genes were located in the scaffolds which could not be assembled in the chromosomes.

Phylogenetic relationships and gene structures analysis of BvARF

To survey the evolutionary relationships between BvARF proteins from sugar beet and previously-reported ARF proteins from the dicot Arabidopsis we constructed an unrooted phylogenetic tree with $16 \mathrm{BvARF}$ and 23 Arabidopsis ARF protein sequences. The results are shown in Fig 4, based on existing descriptions of ARF conserved domains in Arabidopsis (Hagen \& Guilfoyle, 2002), and all 39 ARF proteins were divided into six categories.

The full-length sequence of the 16 BvARF proteins screened by MEGA7 was used to make a rootless tree by the neighbor-joining method, and the bootstrap procedure was tested with 1000 bootstrap repetitions. The results are shown in Fig 5, which are also divided into six categories: Class I (AtARF1/2-like), Class II (AtARF3/4-like), Class III (AtARF5-like), Class IV (AtARF7/19-like), Class V (AtARF6/8-like) and Class VI ( AtARF10/16/17-like). Four members were assigned to Class I (qzmp, orwr, kddw and yzaj) 2 to Class II (opag and efdx), Class III only contains hgze, 2 to Class IV (qfwi and zzhs), 2 to Class V (okdq and gcik), and 3 
to Class VI (ghtj, jrpi and eqms). The intron/exon structure analysis of the BvARF genes can provide valuble imformation concerning evolutionary relationships among taxa. Analysis of these sequences revealed that all BvARF genes contained introns in the coding sequence, and the number of exons varied from 3 to 15 . In general, most of the BvARF members within a given group possessed a similar exon/intron structure in terms of intron numbers and exon length.

Motifs analysis and subcellular localization prediction of BvARF proteins

For the motifs analysis of the BvARF proteins, the expected number of motifs was set to 20 . The motifs were sorted according to the e-value from small to large, and the protein sequences were ranked according to the reliability of the predicted motifs. The results are shown in Fig 6 and Fig 7. Some motifs were common to all BvARF proteins, such as motifs 1, 2, 3, and 5, and some motifs were specific to certain proteins, such as motifs 13, 18, and 20. Comparing the results of the motifs analysis with the conserved domains of the previous Pfam, it was found that the motif 1, 2 constituted the B3 domain, the motif 6, 8, 11, 12 constituted AUX_RESP, and the motif 4, 9, 14 constituted AUX/IAA. Previous studies have shown that ARF activates or inhibits target genes in relation to amino acids in the middle region (Tiwari, 2003; Ulmasov, Hagen \& Guilfoyle, 1999), and it is worth noting that phantom 17 has a dense glutamine (Q) composition. It has shown in previous research that when glutamines gather in the middle region of the ARF proteins were generally considered to have activation domain activity (Shen et al., 2015). It plays a transcriptional activation role in the transcriptional regulation of auxin, and further predicts that qfwi, zzhs, okdq, gcik may have transcriptional activation which were all in Class IV and Class $\mathrm{V}$.

The subcellular localization prediction of CELLO on BvARF proteins is shown in Tab 4. The results showed that the most of BvARF proteins were only scored in the nucleus, that is, they were all more reliably located in the nucleus. The scores of hgze and jrpi were high in cytoplasm and nucleus. Subcellular localization results indicate that almost all BvARF proteins play a regulatory role in the nucleus. 
205

206

207

208

209

210

211

212

213

214

215

216

217

218

219

220

221

222

223

224

225

226

227

228

229

Gene Ontology Annotation

To survey the functions of the BvARF, GO annotation was obtained from PlantTFDB to construct GO graphs. As shown in Fig 8, all BvARF $(14,100 \%)$ are involved in cell part, cell and organelle. The results also showed that the BvARF were involved in diverse biological processes and predominantly participated in metabolic process, biological regulation, regulation of biological process, cellular process $(12,85.7 \%)$, binding $(11,78.6 \%)$ and response to stimulus $(8,57.1 \%)$. The GO terminologies of BvARF were relatively concentrated.

Expression profiles of BvARF genes in sugar beet under normal growth conditions and in response to salinity stress

In recent years, it has reported that auxin acts as integral part of plant response to salinity stress. Thus we compare the levels of expression of BvARF in condition with and without salt treatment. Expression patterns of 14 BvARF in leaves and root were analyzed by CFX96 RealTime System to clarify the roles of BvARF genes in salt tolerance. The results are shown in Fig 9 and Fig 10. In the leaves, except for jrpi, yzaj and eqms, other BvARF genes all showed different degrees of up-regulation or down-regulation. Most of them showed a down-regulation, and in which the degree of 6 BvARF were significantly reduced (ghtj, okdq, opag, zzhs and efdx). And a few BvARF genes were up-regulated, with only upward trend of gcik was obvious. In the root, after treatment with salinity stress, except for qfwi, orwr and eqms, other BvARF genes all showed different degrees of up-regulation or down-regulation. Most of the BvARF genes showed an upward trend, and in which 6 BvARF showed an obvious upward trend (hgze, okdq, yzaj, kddw, gcik and efdx). And 5 BvARF genes showed a downward trend, but the degree of down-regulation was not significant (ghtj, qzmp, qfwi, jrpi and eqms).

\section{Discussion}

Sugar beet is mainly distributed in Northwest, Northeast and North China, where the salinealkali soil area is larger. Therefore, it is particularly important to study the salt tolerance 
230

231

232

233

234

235

236

237

238

239

240

241

242

243

244

245

246

247

248

249

250

251

252

253

254

255

mechanism of sugar beet and cultivate new varieties. Members of the ARF family have been reported to be involved in regulation of plant growth, development and response to abiotic stresses. However, the ARF family members in sugar beet have not been studied previously. Thus in order to shed light on the roles of BvARF genes in response salt stress, we delineated the major structural characteristics and expression profiles of ARF genes in this species.

In this study, 16 BvARF proteins were discovered by genome-wide analysis, which were transcribed by 14 BvARF genes. The number of members of the BvARF gene family was far less than which of Arabidopsis(23), maize(31), rice(25) and other plants. The lack of clustering in spatial locations indicates that there is less gene duplication in the BvARF gene in the longterm evolution process (Cannon et al., 2004; Tang et al., 2018).

The phylogenetic comparison of ARF proteins has been widely used in many species, and these proteins have been widely reported in different species and evolutionary relationships. In this paper, according to the phylogenetic tree analysis, BvARF proteins and AtARF were divided into six categories. Four BvARF proteins with similar gene structures belong to the Class I ARF, whereas 13 AtARFs were assigned to this group. This suggests that the genes of the group may have been either lost from the sugar beet lineage or acquired in the Arabidopsis after divergence from the last common ancestor shared by Arabidopsis and sugar beet. And Arabidopsis I ARFlike is closely related to the development of flower and seedling, suggesting that these four ARF may have similar functions in beta vulgaris (Ellis et al., 2005). Three BvARF proteins belong to Class II ARF, and according to the Class II ARF function in Arabidopsis, which can affect the dorsal ventrality of lateral organs (Pekker, Alvarez \& Eshed, 2005). One BvARF protein belongs to Class III ARF, suggesting that it may play a regulatory role in stomatal development and petal differentiation(Cole et al., 2009). Two BvARF proteins belong to Class IV ARF and may regulate lateral roots (Okushima et al., 2005). Three BvARF proteins belong to Class V and may be involved in the regulation of auxin homeostasis (Tian et al., 2004). Three BvARF proteins belonging to Class VI may regulate the formation of pollen walls (Wang et al., 2017). 
256

257

258

259

260

261

262

263

264

265

266

267

268

269

270

271

272

273

274

275

276

277

278

279

280

281

282

The localization of all 16 BvARF proteins was predicted in the nucleus, indicating their roles in transcriptional regulation. All 16 BvARF proteins have both a B3 DNA binding domain and an ARF response domain, moreover 5 BvARF proteins lack of a CTD which allows dimerization of the ARF proteins and the Aux/IAA proteins. The percentage of members missing CTD $(31.3 \%)$ was similar to other species, such as tea trees $(26.7 \%)$ (Xu, Mao, Chen, Qian, Liu, Hao, Li \& Chen, 2016)and tomatoes (28.6\%)(Kumar, Tyagi \& Sharma, 2011).

The exon intron pattern shows the homology between species to some extent. The exonintron splicing arrangement and intron numbers in the BvARF genes in the sugar beet genome were similar to other plants. It is worth noting that BvARF genes in Class VI is consistent with the AtARF genes in Class VI, and the exons are long and are less separated by introns. Motif analysis indicated that the motif $1,2,4,6,8,9,11,12$ and 14 present in most of the BvARF proteins, are the typical motifs present in the auxin response factors protein. Motif 1 and 2 are involved in DNA binding, while 4, 9 and 14 are involved in protein-protein interaction. ARF in a group often has a common motif belonging to their own group. Generally, most of the BvARF genes in one group had similar gene structure and conserved motifs, which further supports their classification as described here and the evolutionary relationships among the groups. Previous studies have shown that the regulation of ARF activation or inhibition is mainly determined by the type of amino acids in the middle region. Belong to Class IV and V, four BvARF (qfwi, zzhs, okdq, gcik) have a poly-Q motif in the central region, indicating that they have an activation effect.

$\mathrm{ARF}$ is a kind of transcription factor that regulates the expression of auxin-responsive genes. The expression of ARF is also affected by biological and abiotic stresses (Hannah, Heyer \& Hincha, 2005; Jain \& Khurana, 2009; Kang et al., 2018; Yu et al., 2017). Quantitative results of BvARF genes under salt stress allowed us to identify BvARF genes involved in stress response. The results suggested that six of the $14 \mathrm{BvARF}$ genes were induced or inhibited by salt in leaves and six were induced in roots. And some BvARF expressed differently in leaves and roots, such as okdq and efdx, while some showed the same trend, such as gcik. It can be speculated that 
283

284

285

286

287

288

289

290

291

292

293

294

295

296

297

298

299

300

301

302

303

304

305

306

307

308

309

310

311

312

313

314

315

ARF have played different degrees of regulation on the salt tolerance of sugar beet. Our study provides evidence that BvARF may participate in sugar beet response to salt stress. Further research is needed to determine which mechanism to achieve salt tolerance.

\section{Conclusion}

In this study, a total of 16 BvARF protein were identified, and we analyzed the gene structures, chromosome location, protein conserved domains, phylogeny, proteins motif, subcellular localization and gene ontology consortium etc. by bioinformatics method. And quantitatively comparing the expression of BvARF in leaves and roots under salt stress and normal environment. This paper laid a foundation for further study of the functional characteristics of BvARF, and provided a new idea for breeding salt-tolerant sugar beet varieties.

\section{References}

Bailey TL, Williams N, Misleh C, and Li WW. 2006. MEME: discovering and analyzing DNA and protein sequence motifs. Nucleic Acids Res 34:W369-373. 10.1093/nar/gk1198

Boer DR, Freire-Rios A, van den Berg WA, Saaki T, Manfield IW, Kepinski S, Lopez-Vidrieo I, Franco-Zorrilla JM, de Vries SC, Solano R, Weijers D, and Coll M. 2014. Structural basis for DNA binding specificity by the auxin-dependent ARF transcription factors. Cell 156:577-589. 10.1016/j.cell.2013.12.027

Cannon SB, Mitra A, Baumgarten A, Young ND, and May G. 2004. The roles of segmental and tandem gene duplication in the evolution of large gene families in Arabidopsis thaliana. BMC Plant Biol 4:10. 10.1186/1471-2229-4-10

Chandler JW. 2016. Auxin response factors. Plant Cell Environ 39:1014-1028. $10.1111 /$ pce. 12662

Cole M, Chandler J, Weijers D, Jacobs B, Comelli P, and Werr W. 2009. DORNROSCHEN is a direct target of the auxin response factor MONOPTEROS in the Arabidopsis embryo. Development 136:1643-1651. 10.1242/dev.032177

Dohm JC, Lange C, Holtgrawe D, Sorensen TR, Borchardt D, Schulz B, Lehrach H, Weisshaar B, and Himmelbauer H. 2012. Palaeohexaploid ancestry for Caryophyllales inferred from extensive gene-based physical and genetic mapping of the sugar beet genome (Beta vulgaris). Plant J 70:528-540. 10.1111/j.1365-313X.2011.04898.x

Dohm JC, Minoche AE, Holtgrawe D, Capella-Gutierrez S, Zakrzewski F, Tafer H, Rupp O, Sorensen TR, Stracke R, Reinhardt R, Goesmann A, Kraft T, Schulz B, Stadler PF, Schmidt T, Gabaldon T, Lehrach H, Weisshaar B, and Himmelbauer H. 2014. The genome of the recently domesticated crop plant sugar beet (Beta vulgaris). Nature 
316

317

318

319

320

321

322

323

324

325

326

327

328

329

330

331

332

333

334

335

336

337

338

339

340

341

342

343

344

345

346

347

348

349

350

351

352

353

354

355

356

505:546-549. 10.1038/nature12817

Ellis CM, Nagpal P, Young JC, Hagen G, Guilfoyle TJ, and Reed JW. 2005. AUXIN RESPONSE FACTOR1 and AUXIN RESPONSE FACTOR2 regulate senescence and floral organ abscission in Arabidopsis thaliana. Development 132:4563-4574. 10.1242/dev.02012

Finn RD, Coggill P, Eberhardt RY, Eddy SR, Mistry J, Mitchell AL, Potter SC, Punta M, Qureshi M, Sangrador-Vegas A, Salazar GA, Tate J, and Bateman A. 2016. The Pfam protein families database: towards a more sustainable future. Nucleic Acids Res 44:D279285. 10.1093/nar/gkv1344

Fleming AJ. 2006. Plant signalling: the inexorable rise of auxin. Trends Cell Biol 16:397-402. 10.1016/j.tcb.2006.06.005

Hagen G, and Guilfoyle T. 2002. Auxin-responsive gene expression: genes, promoters and regulatory factors. Plant Molecular Biology 49:373-385. 10.1023/a:1015207114117

Hannah MA, Heyer AG, and Hincha DK. 2005. A global survey of gene regulation during cold acclimation in Arabidopsis thaliana. PLoS Genet 1:e26. 10.1371/journal.pgen.0010026

$\mathrm{Hu}$ B, Jin J, Guo AY, Zhang H, Luo J, and Gao G. 2015a. GSDS 2.0: an upgraded gene feature visualization server. Bioinformatics 31:1296-1297. 10.1093/bioinformatics/btu817

Hu W, Zuo J, Hou X, Yan Y, Wei Y, Liu J, Li M, Xu B, and Jin Z. 2015b. The auxin response factor gene family in banana: genome-wide identification and expression analyses during development, ripening, and abiotic stress. Front Plant Sci 6:742. 10.3389/fpls.2015.00742

Jain M, and Khurana JP. 2009. Transcript profiling reveals diverse roles of auxin-responsive genes during reproductive development and abiotic stress in rice. Febs $j$ 276:3148-3162. 10.1111/j.1742-4658.2009.07033.x

Kang C, He S, Zhai H, Li R, Zhao N, and Liu Q. 2018. A Sweetpotato Auxin Response Factor Gene (IbARF5) Is Involved in Carotenoid Biosynthesis and Salt and Drought Tolerance in Transgenic Arabidopsis. Front Plant Sci 9:1307. 10.3389/fpls.2018.01307

Krogan NT, Marcos D, Weiner AI, and Berleth T. 2016. The auxin response factor MONOPTEROS controls meristem function and organogenesis in both the shoot and root through the direct regulation of PIN genes. New Phytol 212:42-50. 10.1111/nph.14107

Kumar R, Tyagi AK, and Sharma AK. 2011. Genome-wide analysis of auxin response factor (ARF) gene family from tomato and analysis of their role in flower and fruit development. Mol Genet Genomics 285:245-260. 10.1007/s00438-011-0602-7

Larkin MA, Blackshields G, Brown NP, Chenna R, McGettigan PA, McWilliam H, Valentin F, Wallace IM, Wilm A, Lopez R, Thompson JD, Gibson TJ, and Higgins DG. 2007. Clustal W and Clustal X version 2.0. Bioinformatics 23:2947-2948. 10.1093/bioinformatics/btm404

Li J, Dai X, and Zhao Y. 2006. A role for auxin response factor 19 in auxin and ethylene signaling in Arabidopsis. Plant Physiol 140:899-908. 10.1104/pp.105.070987

Li SB, OuYang WZ, Hou XJ, Xie LL, Hu CG, and Zhang JZ. 2015. Genome-wide identification, isolation and expression analysis of auxin response factor (ARF) gene family in sweet 
orange (Citrus sinensis). Front Plant Sci 6:119. 10.3389/fpls.2015.00119

Li SB, Xie ZZ, Hu CG, and Zhang JZ. 2016. A Review of Auxin Response Factors (ARFs) in Plants. Front Plant Sci 7:47. 10.3389/fpls.2016.00047

Liu X, Dinh TT, Li D, Shi B, Li Y, Cao X, Guo L, Pan Y, Jiao Y, and Chen X. 2014. AUXIN RESPONSE FACTOR 3 integrates the functions of AGAMOUS and APETALA2 in floral meristem determinacy. Plant $J$ 80:629-641. 10.1111/tpj.12658

Ljung K. 2013. Auxin metabolism and homeostasis during plant development. Development 140:943-950. 10.1242/dev.086363

Okushima Y, Overvoorde PJ, Arima K, Alonso JM, Chan A, Chang C, Ecker JR, Hughes B, Lui A, Nguyen D, Onodera C, Quach H, Smith A, Yu G, and Theologis A. 2005. Functional genomic analysis of the AUXIN RESPONSE FACTOR gene family members in Arabidopsis thaliana: unique and overlapping functions of ARF7 and ARF19. Plant Cell 17:444-463. 10.1105/tpc. 104.028316

Pekker I, Alvarez JP, and Eshed Y. 2005. Auxin response factors mediate Arabidopsis organ asymmetry via modulation of KANADI activity. Plant Cell 17:2899-2910. $10.1105 /$ tpc. 105.034876

Qi Y, Wang S, Shen C, Zhang S, Chen Y, Xu Y, Liu Y, Wu Y, and Jiang D. 2012. OsARF12, a transcription activator on auxin response gene, regulates root elongation and affects iron accumulation in rice (Oryza sativa). New Phytol 193:109-120. 10.1111/j.14698137.2011.03910.x

Roosjen M, Paque S, and Weijers D. 2018. Auxin Response Factors: output control in auxin biology. J Exp Bot 69:179-188. 10.1093/jxb/erx237

Shen C, Wang S, Zhang S, Xu Y, Qian Q, Qi Y, and Jiang de A. 2013. OsARF16, a transcription factor, is required for auxin and phosphate starvation response in rice (Oryza sativa L.). Plant Cell Environ 36:607-620. 10.1111/pce.12001

Shen C, Yue R, Sun T, Zhang L, Xu L, Tie S, Wang H, and Yang Y. 2015. Genome-wide identification and expression analysis of auxin response factor gene family in Medicago truncatula. Front Plant Sci 6:73. 10.3389/fpls.2015.00073

Shi SZ, Cui J, Lu ZX, Cheng DY, Luo CF. 2008. Salt tolerance screening of Sugarbeet Germplasm Resources. China Beet and Sugar 4:7-9.

$\mathrm{Su}$ YH, Liu YB, Bai B, and Zhang XS. 2014. Establishment of embryonic shoot-root axis is involved in auxin and cytokinin response during Arabidopsis somatic embryogenesis. Front Plant Sci 5:792. 10.3389/fpls.2014.00792

Tang Y, Bao X, Liu K, Wang J, Zhang J, Feng Y, Wang Y, Lin L, Feng J, and Li C. 2018. Genome-wide identification and expression profiling of the auxin response factor (ARF) gene family in physic nut. Plos One 13:e0201024. 10.1371/journal.pone.0201024

Tian CE, Muto H, Higuchi K, Matamura T, Tatematsu K, Koshiba T, and Yamamoto KT. 2004. Disruption and overexpression of auxin response factor 8 gene of Arabidopsis affect hypocotyl elongation and root growth habit, indicating its possible involvement in auxin homeostasis in light condition. Plant J 40:333-343. 10.1111/j.1365-313X.2004.02220.x Tiwari SB. 2003. The Roles of Auxin Response Factor Domains in Auxin-Responsive 
Transcription. The Plant Cell Online 15:533-543. 10.1105/tpc.008417

Ulmasov T, Hagen G, and Guilfoyle TJ. 1997. ARF1, a transcription factor that binds to auxin response elements.

Ulmasov T, Hagen G, and Guilfoyle TJ. 1999. Activation and repression of transcription by auxin-response factors. Proceedings of the National Academy of Sciences 96:5844-5849.

Wan S, Li W, Zhu Y, Liu Z, Huang W, and Zhan J. 2014. Genome-wide identification, characterization and expression analysis of the auxin response factor gene family in Vitis vinifera. Plant Cell Rep 33:1365-1375. 10.1007/s00299-014-1622-7

Wang B, Xue JS, Yu YH, Liu SQ, Zhang JX, Yao XZ, Liu ZX, Xu XF, and Yang ZN. 2017. Fine regulation of ARF17 for anther development and pollen formation. BMC Plant Biol 17:243. 10.1186/s12870-017-1185-1

Wang D, Pei K, Fu Y, Sun Z, Li S, Liu H, Tang K, Han B, and Tao Y. 2007. Genome-wide analysis of the auxin response factors (ARF) gene family in rice (Oryza sativa). Gene 394:13-24. 10.1016/j.gene.2007.01.006

Xing H, Pudake RN, Guo G, Xing G, Hu Z, Zhang Y, Sun Q, and Ni Z. 2011. Genome-wide identification and expression profiling of auxin response factor (ARF) gene family in maize. BMC Genomics 12:178. 10.1186/1471-2164-12-178

Xu YX, Mao J, Chen W, Qian TT, Liu SC, Hao WJ, Li CF, and Chen L. 2016. Identification and expression profiling of the auxin response factors (ARFs) in the tea plant (Camellia sinensis (L.) O. Kuntze) under various abiotic stresses. Plant Physiol Biochem 98:46-56. 10.1016/j.plaphy.2015.11.014

Yu C, Zhan Y, Feng X, Huang ZA, and Sun C. 2017. Identification and Expression Profiling of the Auxin Response Factors in Capsicum annuum L. under Abiotic Stress and Hormone Treatments. Int J Mol Sci 18. 10.3390/ijms18122719

Yu H, Soler M, Mila I, San Clemente H, Savelli B, Dunand C, Paiva JA, Myburg AA, Bouzayen M, Grima-Pettenati J, and Cassan-Wang H. 2014. Genome-wide characterization and expression profiling of the AUXIN RESPONSE FACTOR (ARF) gene family in Eucalyptus grandis. Plos One 9:e108906. 10.1371/journal.pone.0108906

Zhang JY, He SB, Li L, and Yang HQ. 2014. Auxin inhibits stomatal development through MONOPTEROS repression of a mobile peptide gene STOMAGEN in mesophyll. Proc Natl Acad Sci U S A 111:E3015-3023. 10.1073/pnas.1400542111

Zhao S, Zhang ML, Ma TL, and Wang Y. 2016. Phosphorylation of ARF2 Relieves Its Repression of Transcription of the K+ Transporter Gene HAK5 in Response to Low Potassium Stress. Plant Cell 28:3005-3019. 10.1105/tpc.16.00684 
Figure 1

Conservative domains analysis of BvARF.

In the figure, the green color block on the left represents the B3 domain, the middle red color block represents the AUX_RESP domain, and the orange color block on the right represents the AUX/IAA domain.

ghtj

qzmp

hgze

qfwi

jrpi

okdq

yzaj

orwr

kddw

eqms

opag

zzhs

gcik.t1

gcik.t2

efdx.t1

efdx.t2

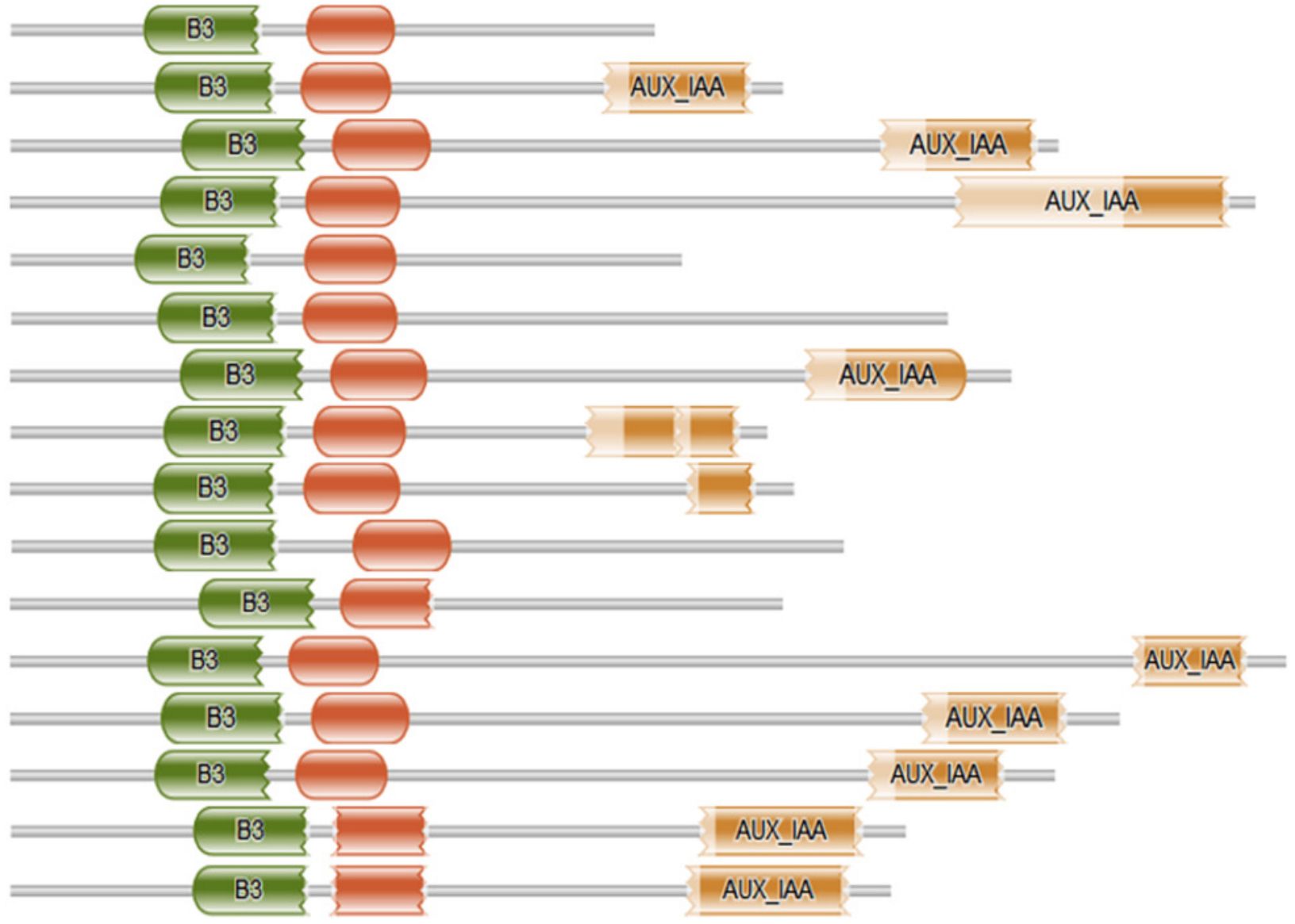


Figure 2

Conserved domain of BvARF proteins.

A.alignment result of B3 domain. B.weblogo of B3 domain. C.alignment result of AUX_RESP domain. D.weblogo of AUX_RESP domain E.alignment result of AUX/IAA domain. F.weblogo of AUX/IAA domain. 

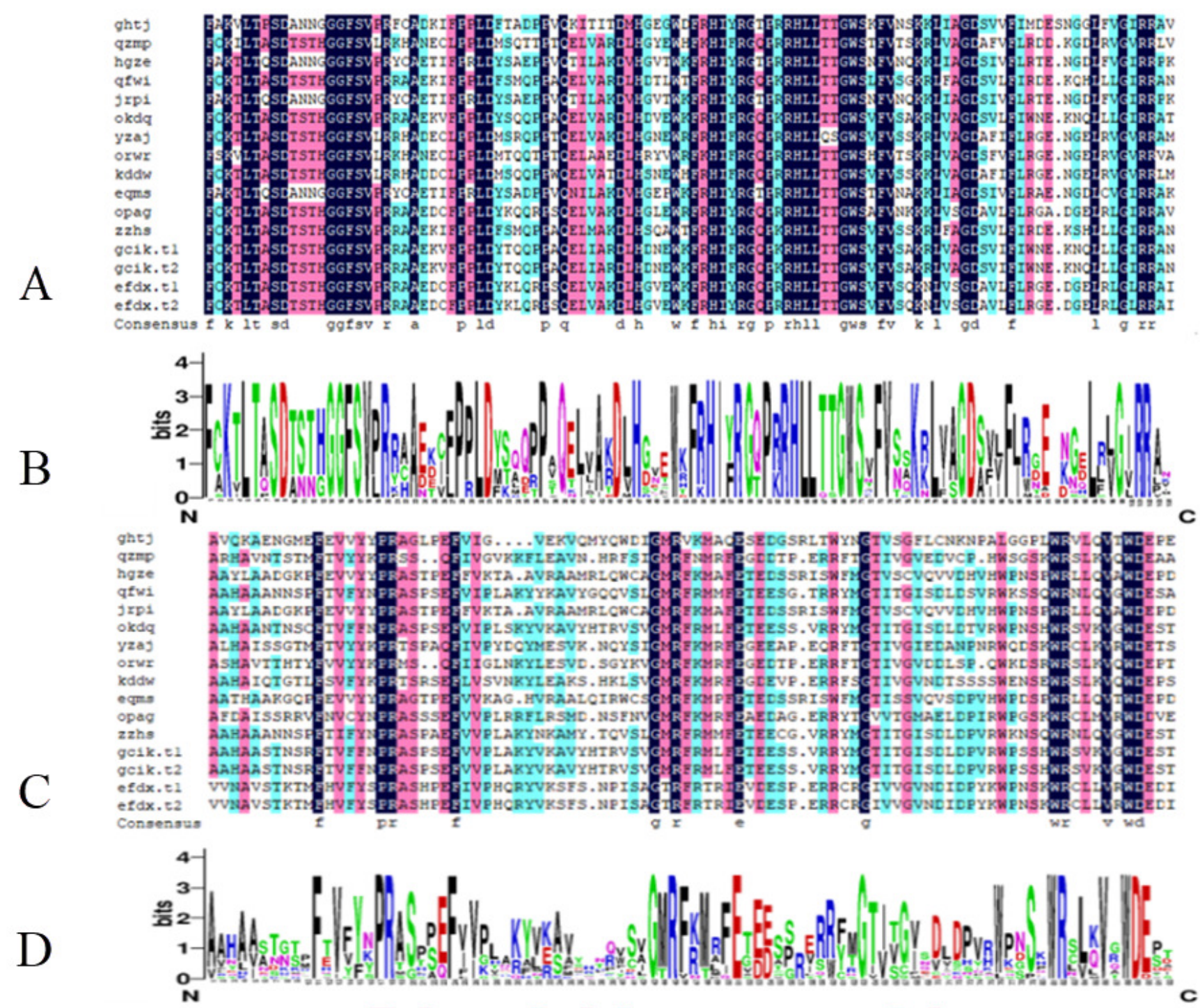

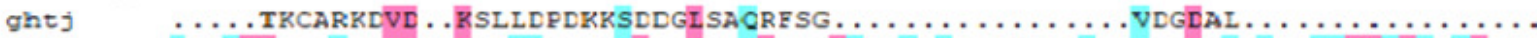

qZMP RSRTKVHMCGVAVG. .RAVDLTIIEGȲDEIISEIERMFDIKGELOTRN . KWAVVFTDDEGDMMLVGLDEWCEECKM

hgze $\quad \ldots$ CKVFMESEDVG . NIILSLSSIGTYDGIYKKIAHHIWCG $\ldots \ldots$ K.... IIEAECCALS $\ldots \ldots \ldots \ldots$

qfW1 RTYTKVYRRG.AVG..BSIDVTRYSDYEEIRCDIARMFGIE GCMEDRGRVGWKLVYVDHENDVLLVGDDFWEDFVNC

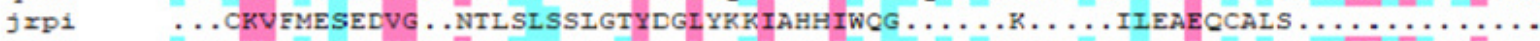

okdq RTYVKVYRSG.SVG.. RSLDISRFSSYHEIREELGCMFGIEGMIDDPLRSGWOLVFVDRENDVLLLGDDFWEAFVYN

YZaj RSCTKVCRCGIALG. .RSVDLSKFSDYDCIVEEI DHIFDFNGELKIPEK. NWLIVYTDDEGDMMLVGDDFWOEECSM

OYWY RSRTKVCMCGVAVG. . RAVDITIIESYDCI IDEIEELFDLKGELRFRD. . KWEIVFTINEGDMMLVGDDFWTEFSSM

kddw .TCTKVHMCDVAVG. . RAVDLTRFECYEDILKKLELIFDIKGELCCSSG. KWCVVYTDDEDDMMMVGDDFWHEFCSM

eqms TGHCRVFMESEDVG. . RTLELTLINSYEEICSRIANMFGIESSDMLNR. . . . . VVYRLSAGSSRCIGDEPFSEFARR

Opag SFPPPETSEWPLVEGCPCYKSSSATKVVSSCPGVNDIYAVRDMLIDIAL $\ldots \ldots \ldots \ldots \ldots \ldots \ldots \ldots$

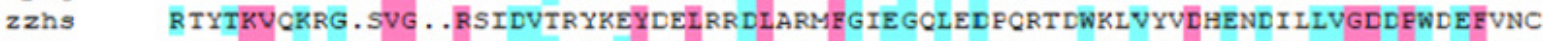

gCik.t 1 GTFVKVCRSG.SLG. .RSLDISRFSSYCEIRSEIAHMEGLEGCIEDPLRSGWQLVFVDRENDVILIGDD FWCEEVNN

gCik.t2 GTFVRVCRSG.SLG. RSLDISRFSSYCEIRSELAHMFGLEGCLEDPLRSGWCLVFVDRENDVLLLGDDPWCEFVNN

F efdx.t1 RSCTKVHRCGSLVG. .RAIDLSRIKSYADIMNEIEHLFGMEGLLCDPAK. GWRIIYTDKENDMMVVGDDPWNEFCDV efdx.t2 RSCTKVHRQGSLVG. RAIDLSKIKSYADIMNELEHIEGME GLIQDPAK. GWRIIYTDKENDMMVVGDDFWNEECDV Consensus

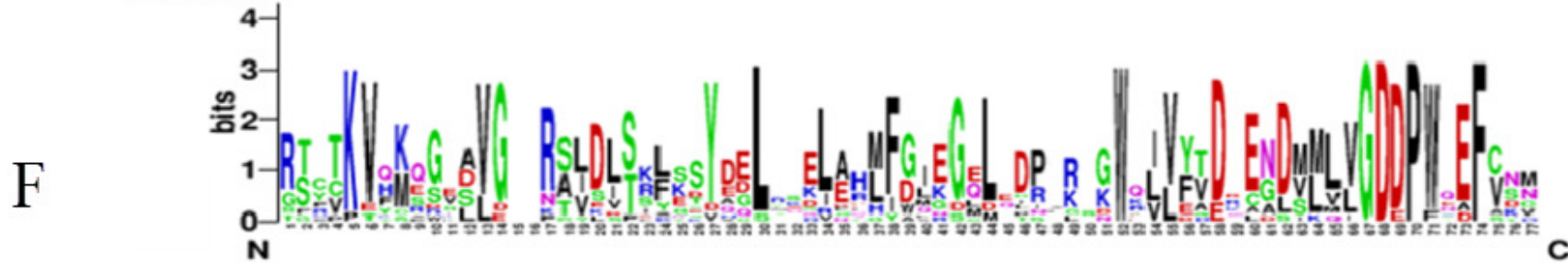


Figure 3

Chromosomal localization of BvARF genes.

The unit of gene position is Mb Phylogenetic relationships and gene structures analysis of BvARF.

Chr 1.map Chr 2.map Chr 3.map Chr 4.map Chr 5.map Chr 6.map Chr 7.map Chr 8.map Chr 9.map
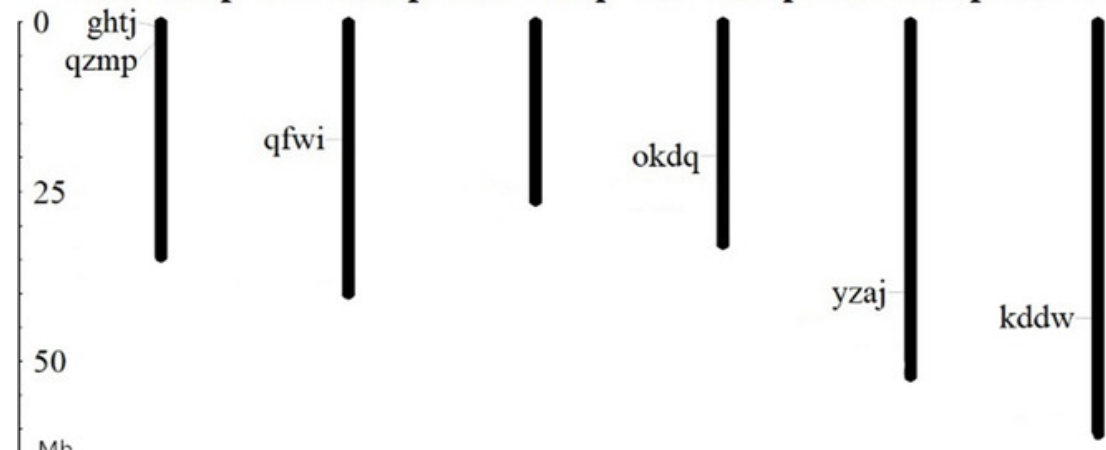

gcik

50

opag

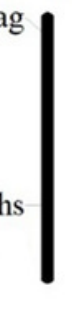

efdx

$\mathrm{Mb}$ 
Figure 4

Phylogenetic relationships of BvARF and AtARF proteins. 


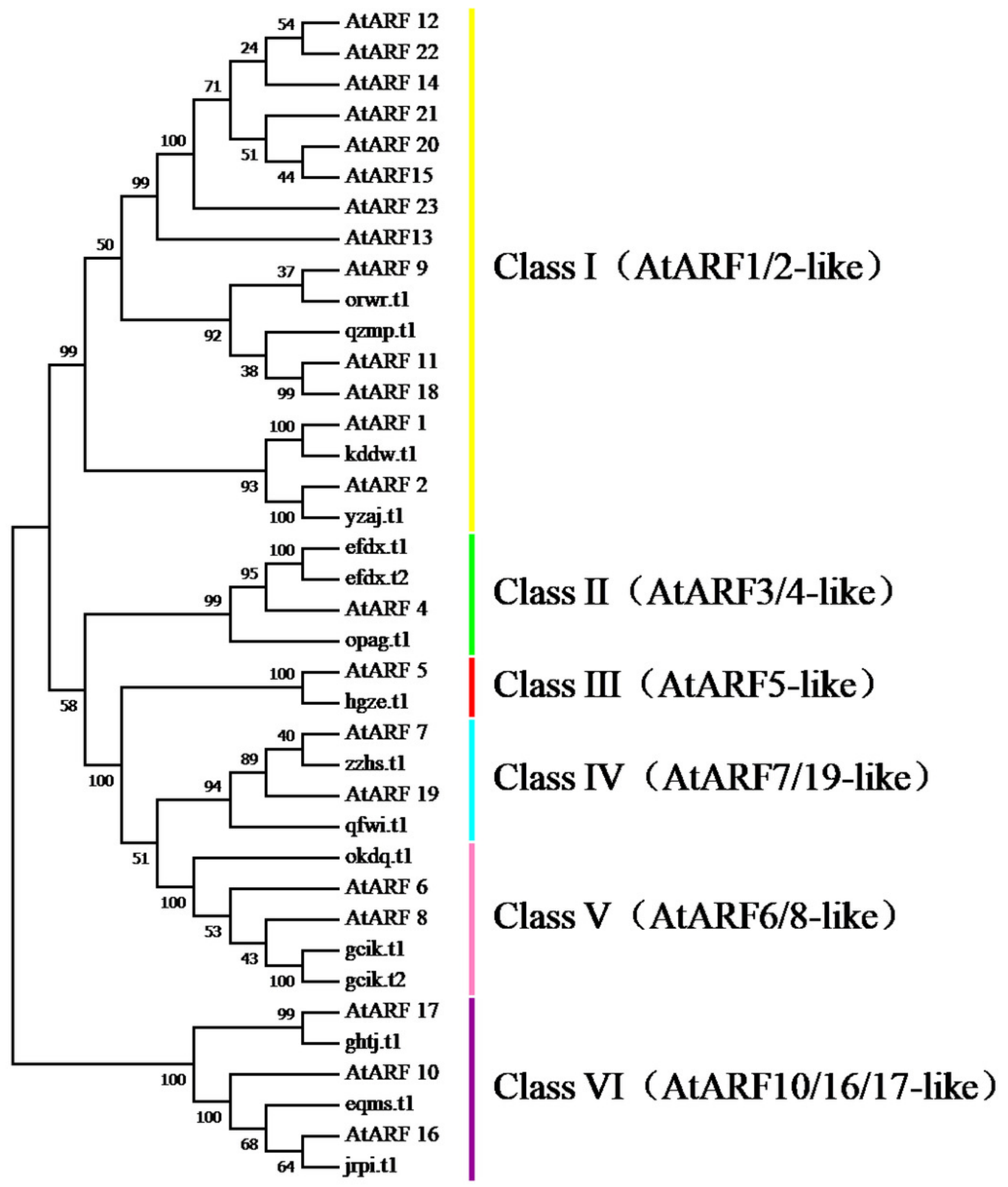




\section{Figure 5}

Phylogenetic relationships of BvARF proteins and exon/intron stuctures of BvARF gene.

Phylogenetic relationships between BvARF proteins. The number on the node represents the confidence value of the branch; the gene Class is represented in a different color on the right side of the rootless tree. Exon/intron stuctures of the BvARF gene. Exons and introns are represented by yellow box and black lines, respectively.

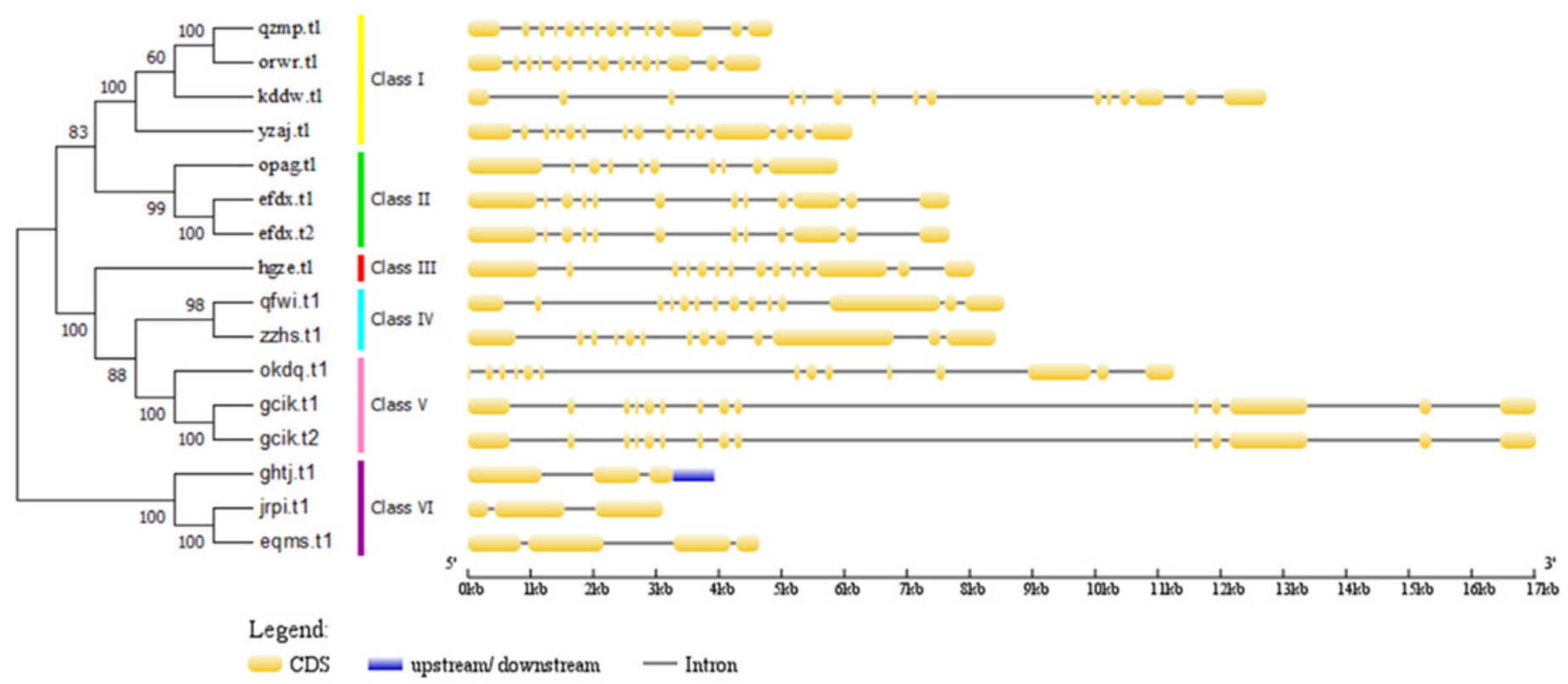




\section{Figure 6}

Motifs in BvARF proteins.

The motifs were arranged according to the e-value from small to large, the letters in each motif were amino abbreviation. The size of the letter represented the saliency of the amino acid in the motif. The larger the letter, the higher the saliency, which is, the higher the frequency at which the amino acid appears in the same position in the same motif in different sequences. 


\section{Motrfs}

Logo

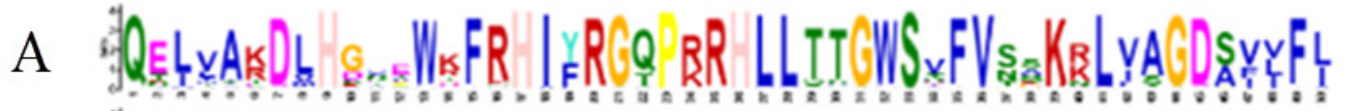

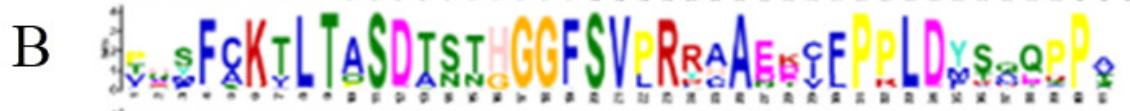
C

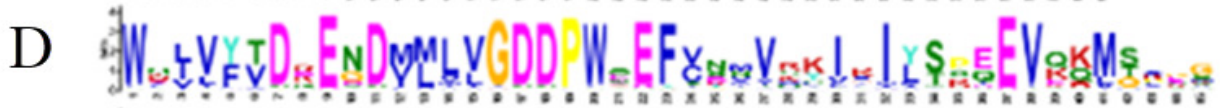
E H.

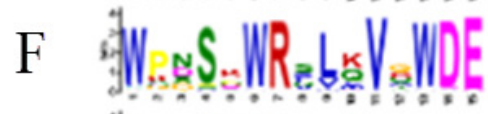
G

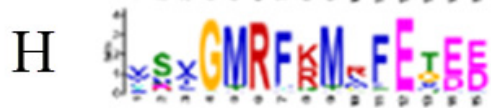
I Hof

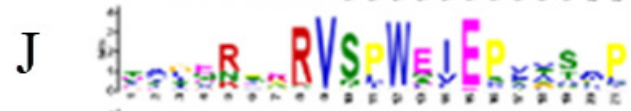

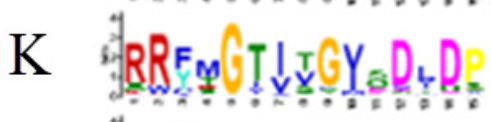

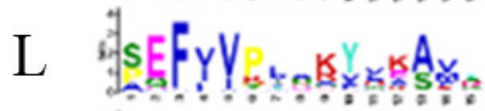

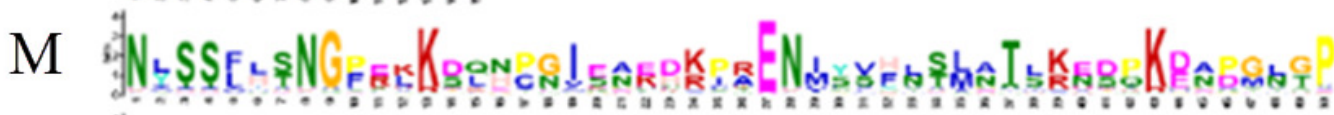

$\mathrm{N}$, prot

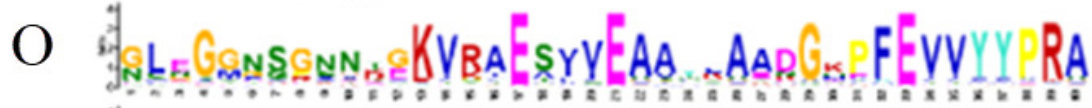

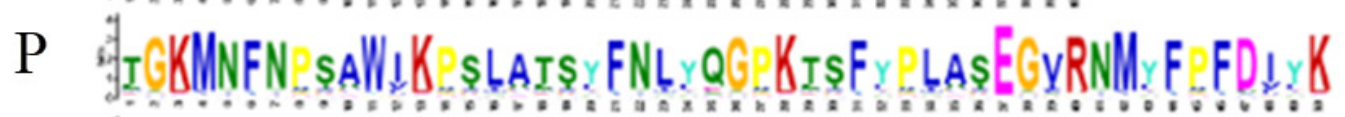

Q

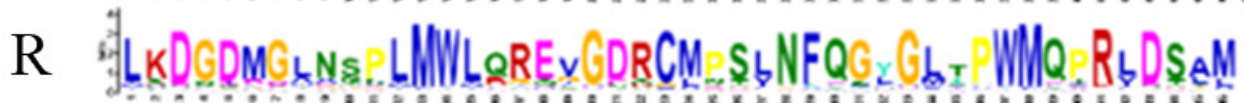

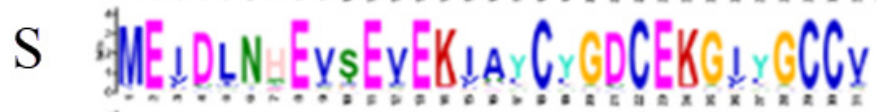

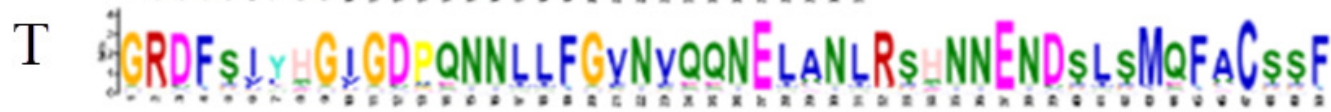


Figure 7

Analysis of BvARF proteins motif.

The different color blocks correspond to different motifs. The width of the color block is the length of the motif. The height of the color block represents the saliency of the motifs in the sequence. The higher the saliency, the more able to match the predicted motifs.

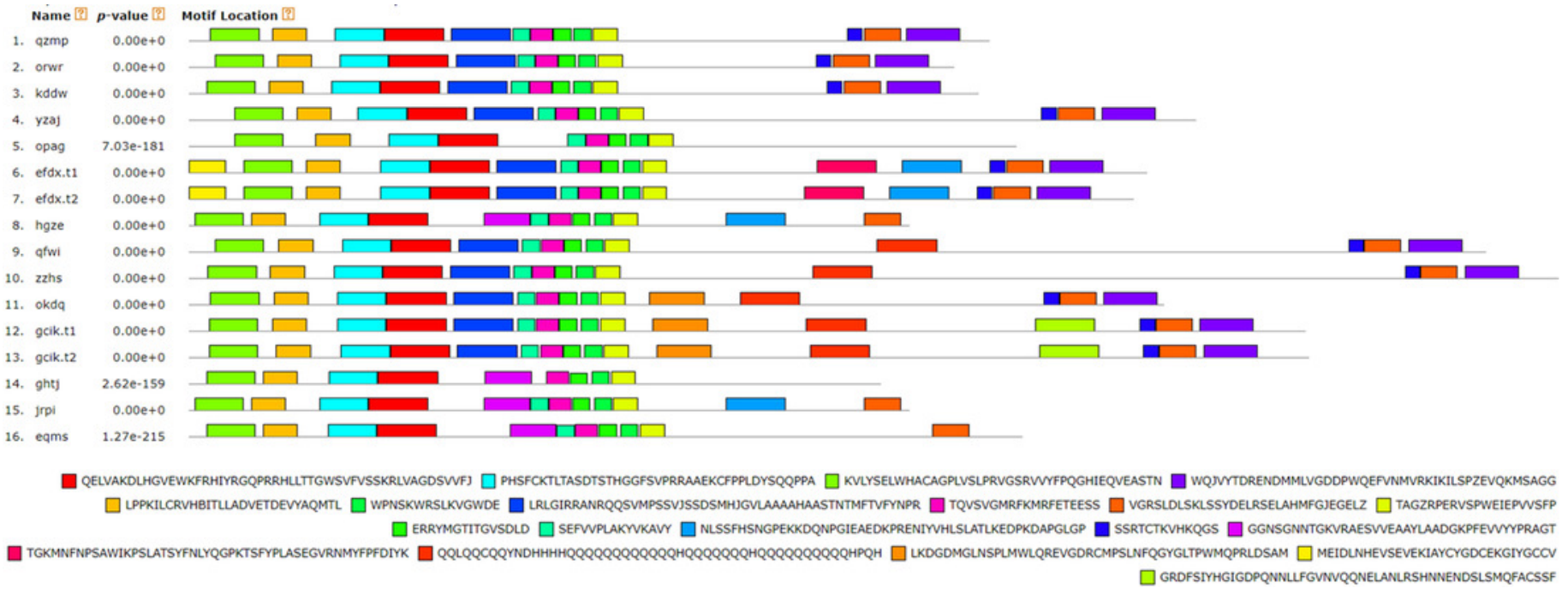


Figure 8

\section{Assignment of GO categories to BvARF genes}

Expression profiles of BvARF genes in sugar beet under normal growth conditions and in response to salinity stress.

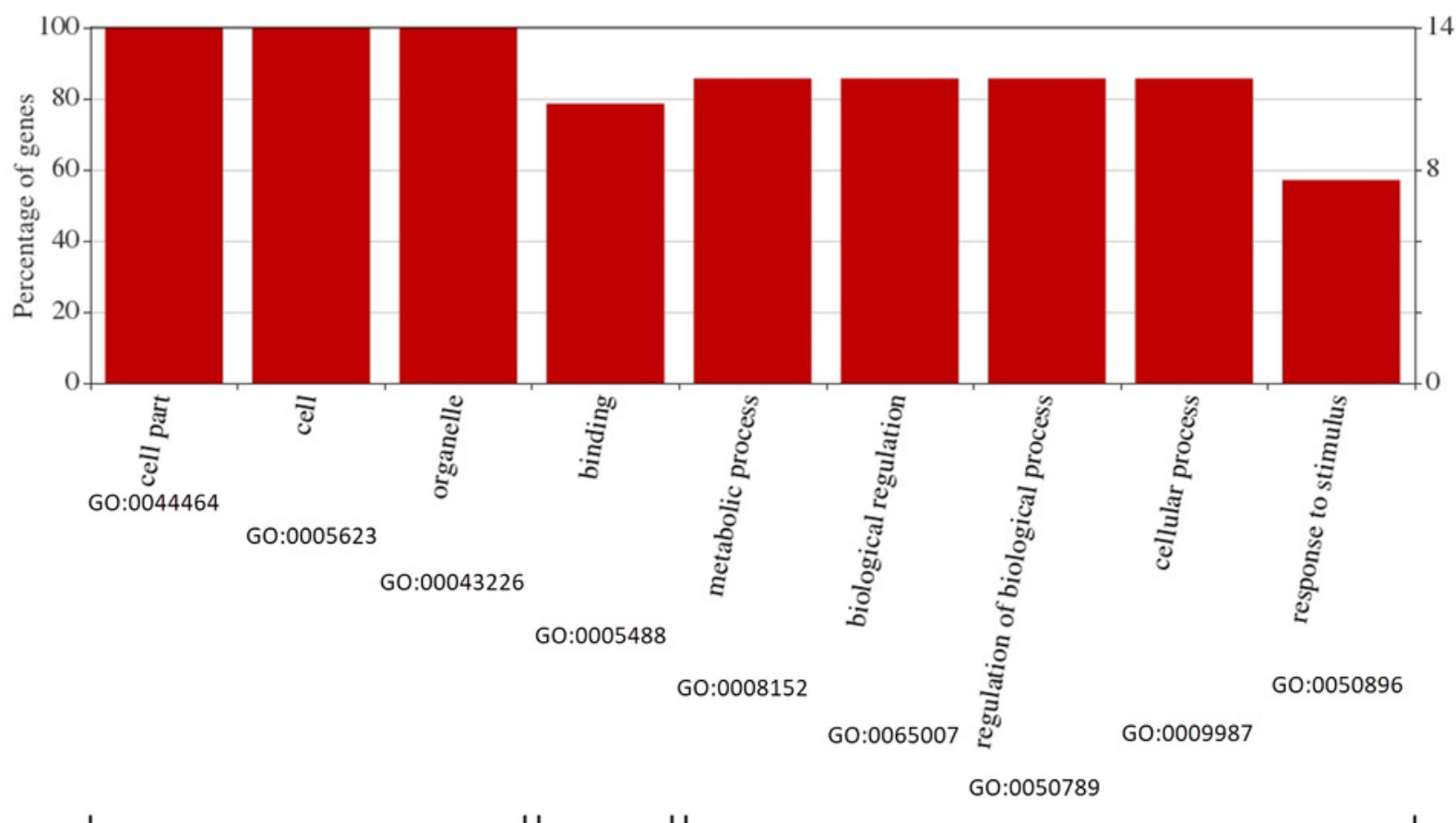


Figure 9

Expression analysis of BARF genes in leaf in response to salinity stress.

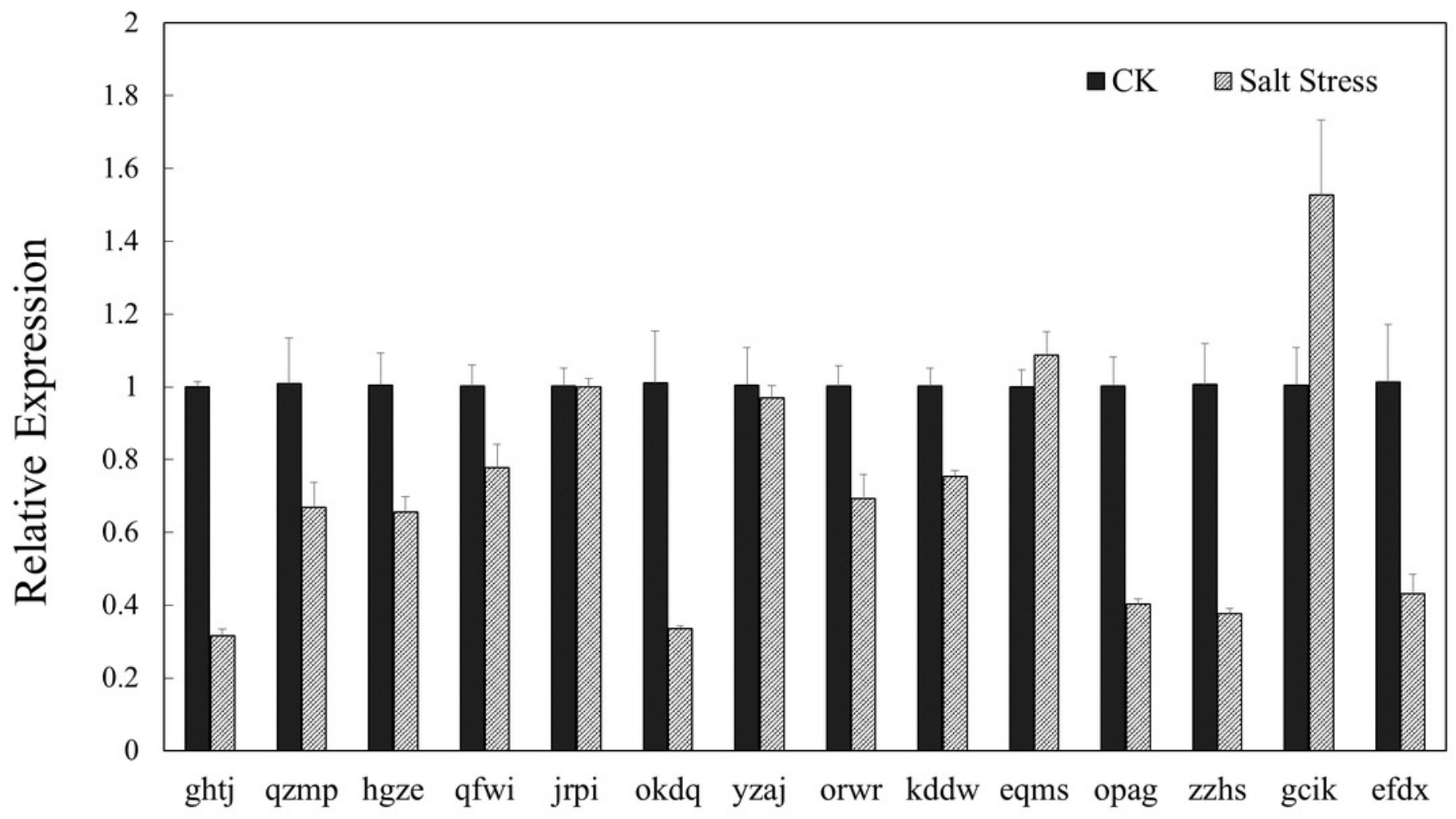


Figure 10

Expression analysis of BvARF genes in root in response to salinity stress.

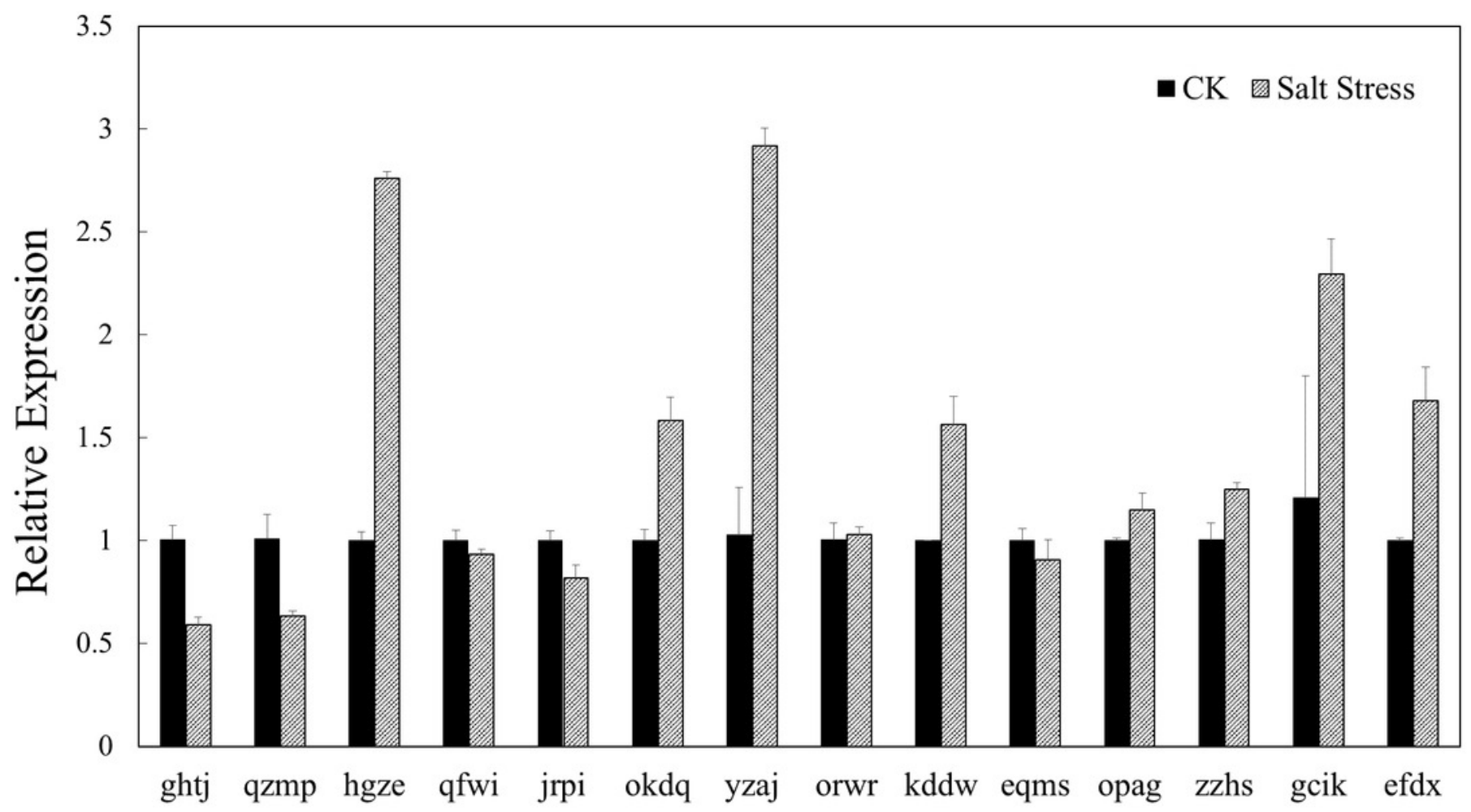


Table $\mathbf{1}$ (on next page)

primer sequences of $A R F$ 
1

\begin{tabular}{|c|c|c|}
\hline Gene & Forward primer( $\left(5^{\prime}-3{ }^{\prime}\right)$ & Reverse primer( $\left.5^{\prime}-3^{\prime}\right)$ \\
\hline ghtj & CTGTGTCCACTGACCTAAA & ATCTCTGAGCACTAAGCCC \\
\hline qzmp & CAAGATTTCTGTAGTCCCG & GTCTCCAGTATTTTGTCCC \\
\hline hgze & GTGTGGCGATAAGCAGAATAG & TCCTTGCCTTTGTTTCCTGTA \\
\hline qfwi & GCTCAGATGACACTCCTACC & AACAAATAGACTCCATCCTG \\
\hline jrpi & TGTTCGTTGGGATTCGGAGG & ATTCCGCTCGCACTTTCTCA \\
\hline okdq & AAGCCTTGTTATCATCCG & GGTTTGCTAGTCCCTCGT \\
\hline yzaj & ATTGAAGGCTGAAGCGGATAC & CGTCTGAGCACCGAGAACC \\
\hline orwr & ATTCAGGTGGAGTTGATGTT & AGGCTTTAGTGGTTCAGTTT \\
\hline kddw & ACGCCACCTACTGACTACA & CCTCATACCAACAGAAAGC \\
\hline eqms & CCGAGTTTGTTGTGAAGGC & GAAGGGGGACAGATGAATG \\
\hline opag & TTCGAGGGTGTCCAAGTTCC & GCGGCAGGAACGGTAGAATA \\
\hline zzhs & AGGGCAACCAAAACGACACT & TCCTAAATCGCATCCCAAGA \\
\hline gcik & GCTTGTATTTGTTGACCG & ACTTCTTGAGGGGATAGG \\
\hline efdx & CGGTATTGTTGTTGGTGTTA & ACATCCATAGGGAGGTGA \\
\hline $\mathrm{ICDH}$ & CACACCAGATGAAGGCCGT & CCCTGAAGACCGTGCCAT \\
\hline
\end{tabular}


Table 2 (on next page)

basic information of BvARF 


\begin{tabular}{llll}
\hline ARF name & NCBI Reference Sequence & Gene ID & Description \\
\hline ghtj & XP_010672559.1 & 1108870177 & PREDICTED: auxin response factor 17 [Beta vulgaris subsp. vulgaris] \\
qzmp & XP_010696146.1 & 1108870906 & PREDICTED: auxin response factor 18 [Beta vulgaris subsp. vulgaris] \\
hgze & XP_010692716.1 & 1108784410 & PREDICTED: auxin response factor 5 [Beta vulgaris subsp. vulgaris] \\
qfwi & XP_010669598.1 & 1108883137 & PREDICTED: auxin response factor 19 [Beta vulgaris subsp. vulgaris] \\
jrpi & XP_010693396.1 & 1108789780 & PREDICTED: auxin response factor 18 isoform X2 [Beta vulgaris subsp. \\
& & & vulgaris] \\
okdq & XP_010675199.1 & 1108911686 & PREDICTED: auxin response factor 8 [Beta vulgaris subsp. vulgaris] \\
yzaj & XP_010678349.1 & 1108924588 & PREDICTED: auxin response factor 2 [Beta vulgaris subsp. vulgaris] \\
orwr & XP_010695583.1 & 1108811087 & PREDICTED: auxin response factor 9 [Beta vulgaris subsp. vulgaris] \\
kddw & XP_010682136.1 & 1108942544 & PREDICTED: auxin response factor 1 [Beta vulgaris subsp. vulgaris] \\
eqms & XP_010684071.1 & 1108952427 & PREDICTED: auxin response factor 18 [Beta vulgaris subsp. vulgaris] \\
opag & XP_010686391.1 & 1108960968 & PREDICTED: auxin response factor 3 isoform X2 [Beta vulgaris subsp. vulgaris] \\
zzhs & XP_010687536.1 & 1108964617 & PREDICTED: auxin response factor 7 isoform X1 [Beta vulgaris subsp. vulgaris] \\
gcik.t1 & XP_010688802.1 & 1108976183 & PREDICTED: auxin response factor 6 isoform X2 [Beta vulgaris subsp. vulgaris] \\
gcik.t2 & XP_010688801.1 & 1108976178 & PREDICTED: auxin response factor 6 isoform X1 [Beta vulgaris subsp. vulgaris] \\
efdx.t1 & XP_010689575.1 & 1108976862 & PREDICTED: auxin response factor 4 isoform X1 [Beta vulgaris subsp. vulgaris] \\
efdx.t2 & XP_010689577.1 & 1108976872 & PREDICTED: auxin response factor 4 isoform X3 [Beta vulgaris subsp. vulgaris] \\
\hline
\end{tabular}




\section{Table 3(on next page)}

Alignment result of BvARF proteins with NCBI non-redundant protein sequences database 
1

\begin{tabular}{ccccc}
\hline ARF name & ORF(bp) & Amino acid & Molecular weight (Da) & PI \\
\hline ghtj & 1734 & 577 & 64060.51 & 6 \\
qzmp & 2007 & 668 & 74885.18 & 6.23 \\
hgze & 2631 & 876 & 97074.28 & 5.21 \\
qfwi & 3249 & 1082 & 119552.61 & 6.19 \\
jrpi & 1806 & 601 & 67120.47 & 7.9 \\
okdq & 2442 & 813 & 90362.34 & 5.92 \\
yzaj & 2523 & 840 & 94146.56 & 6.33 \\
orwr & 1917 & 638 & 71317.15 & 5.47 \\
kddw & 1980 & 659 & 73156.89 & 5.68 \\
eqms & 2088 & 695 & 76649.34 & 6.28 \\
opag & 2073 & 690 & 76782.42 & 6.57 \\
zzhs & 3429 & 1142 & 127711.63 & 6.13 \\
gcik.t1 & 2796 & 931 & 104483.1 & 6.03 \\
gcik.t2 & 2805 & 934 & 104827.53 & 6.03 \\
efdx.t1 & 2400 & 799 & 89067.37 & 6.04 \\
efdx.t2 & 2367 & 788 & 88000.25 & 6.11 \\
\hline
\end{tabular}

2 


\section{Table 4(on next page)}

Subcellular localization of BvARF proteins.

* Confidence score is significantly higher than other, which means higher credibility. 


\begin{tabular}{|c|c|c|c|c|c|c|c|c|c|c|c|c|}
\hline \multirow{3}{*}{$\begin{array}{l}\text { BvARF } \\
\text { proteins }\end{array}$} & \multicolumn{12}{|c|}{ prediction scores } \\
\hline & extracellula & plasma & cytoplasmi & Cytoskeleta & ER & golgi & Lysosoma & mitochondria & chloroplas & peroxisoma & Vacuol & nuclea \\
\hline & $\mathrm{r}$ & membrane & $\mathrm{c}$ & 1 & & & 1 & 1 & $\mathrm{t}$ & 1 & $\mathrm{e}$ & $\mathrm{r}$ \\
\hline \multirow[t]{2}{*}{ qzmp } & 0.067 & 0.073 & 0.739 & 0.011 & 0.02 & 0.06 & 0.015 & 0.124 & 0.179 & 0.032 & 0.023 & $3.652^{*}$ \\
\hline & & & & & 6 & & & & & & & \\
\hline \multirow[t]{2}{*}{ orwr } & 0.032 & 0.027 & 0.571 & 0.007 & 0.01 & 0.04 & 0.004 & 0.088 & 0.159 & 0.018 & 0.005 & $4.028^{*}$ \\
\hline & & & & & 2 & 9 & & & & & & \\
\hline \multirow[t]{2}{*}{ kddw } & 0.074 & 0.044 & 0.404 & 0.014 & 0.05 & 0.07 & 0.016 & 0.158 & 0.354 & 0.039 & 0.027 & $3.744 *$ \\
\hline & & & & & 1 & 5 & & & & & & \\
\hline \multirow[t]{2}{*}{ yzaj } & 0.128 & 0.048 & 0.561 & 0.019 & 0.01 & 0.02 & 0.008 & 0.145 & 0.056 & 0.044 & 0.009 & $3.945^{*}$ \\
\hline & & & & & 2 & 6 & & & & & & \\
\hline \multirow[t]{2}{*}{ opag } & 0.094 & 0.076 & 1.135 & 0.064 & 0.02 & 0.02 & 0.044 & 0.164 & 0.507 & 0.112 & 0.03 & $2.726^{*}$ \\
\hline & & & & & 4 & 4 & & & & & & \\
\hline \multirow[t]{2}{*}{ efdx.t1 } & 0.15 & 0.073 & 0.995 & 0.022 & 0.03 & 0.03 & 0.042 & 0.232 & 0.281 & 0.091 & 0.022 & $3.024^{*}$ \\
\hline & & & & & & 8 & & & & & & \\
\hline \multirow[t]{2}{*}{ efdx.t2 } & 0.148 & 0.078 & 0.906 & 0.024 & 0.02 & 0.03 & 0.046 & 0.302 & 0.313 & 0.071 & 0.022 & $3.022 *$ \\
\hline & & & & & 9 & 8 & & & & & & \\
\hline \multirow[t]{2}{*}{ hgze } & 0.143 & 0.248 & $1.590 *$ & 0.016 & 0.03 & 0.09 & 0.101 & 0.54 & 0.842 & 0.179 & 0.064 & $1.153^{*}$ \\
\hline & & & & & 3 & 2 & & & & & & \\
\hline \multirow[t]{2}{*}{ qfwi } & 0.106 & 0.369 & 0.2 & 0.03 & 0.01 & 0.00 & 0.008 & 0.027 & 0.012 & 0.008 & 0.009 & $4.207^{*}$ \\
\hline & & & & & 6 & 9 & & & & & & \\
\hline \multirow[t]{2}{*}{ zzhs } & 0.219 & 0.811 & 0.304 & 0.046 & 0.05 & 0.01 & 0.016 & 0.059 & 0.032 & 0.024 & 0.015 & $3.409 *$ \\
\hline & & & & & 2 & 4 & & & & & & \\
\hline \multirow[t]{2}{*}{ okdq } & 0.285 & 0.347 & 0.611 & 0.016 & 0.02 & 0.02 & 0.087 & 0.07 & 0.042 & 0.044 & 0.019 & 3.430 \\
\hline & & & & & 7 & 1 & & & & & & * \\
\hline \multirow[t]{2}{*}{ gcik.t1 } & 0.175 & 0.388 & 0.423 & 0.021 & 0.03 & 0.01 & 0.029 & 0.054 & 0.021 & 0.02 & 0.011 & 3.803 \\
\hline & & & & & 6 & 8 & & & & & & * \\
\hline gcik.t2 & 0.197 & 0.401 & 0.411 & 0.02 & 0.03 & 0.01 & 0.032 & 0.051 & 0.021 & 0.02 & 0.011 & 3.782 \\
\hline
\end{tabular}




\begin{tabular}{|c|c|c|c|c|c|c|c|c|c|c|c|c|}
\hline & & & & & 5 & 9 & & & & & & $*$ \\
\hline \multirow[t]{2}{*}{ ghtj } & 0.131 & 0.122 & 1.376 & 0.016 & 0.02 & 0.03 & 0.074 & 0.212 & 0.64 & 0.129 & 0.064 & 2.184 \\
\hline & & & & & & 2 & & & & & & $*$ \\
\hline \multirow[t]{2}{*}{ jrpi } & 0.143 & 0.248 & $1.590^{*}$ & 0.016 & 0.03 & 0.09 & 0.101 & 0.54 & 0.842 & 0.179 & 0.064 & 1.153 \\
\hline & & & & & 3 & 2 & & & & & & $*$ \\
\hline \multirow[t]{2}{*}{ eqms } & 0.17 & 0.117 & 1.049 & 0.011 & 0.04 & 0.03 & 0.047 & 0.163 & 0.447 & 0.042 & 0.021 & 2.862 \\
\hline & & & & & 1 & 2 & & & & & & $*$ \\
\hline
\end{tabular}

* Confidence score is significantly higher than other, which means higher credibility. 